\section{Stress fördert Schlaganfälle}

Je stärker ältere Menschen unter Stress stehen, desto höher ist ihr Risiko, einen tödlichen hämorrhagischen Schlaganfall zu erleiden, belegt eine US-Studie an 4120 Bürgern im Alter von 65 Jahren aufwärts. Senioren mit dem höchsten Stressniveau hatten ein fast dreimal so hohes Risiko wie Personen mit der geringsten psychischen Belastung. Ausgewertet wurden depressive Symptome, subjektiver Stress, Neurotizismus sowie allgemeine Unzufriedenheit.

Henderson Ket al, Stroke 2013; online 13.12.2012

\section{Früher Schlaf macht gute Noten}

Wer vor Prüfungen zeitig ins Bett geht, hat bessere Noten. In einer Studie aus Edinburgh wurde bei 31 Medizinstudenten anhand eines Schlaftagebuchs der Chronotyp ermittelt und nach Befragung zu Abitur- und Physikumsnoten analysiert, inwieweit dieser mit den Noten korrelierte: Ein Zusammenhang zwischen Chronotyp und Noten bestand nicht - allerdings zwischen dem Zeitpunkt des Zubettgehens in den zwei Wochen vor der Prüfung und der Note. Wer vor der Prüfung früher schlafen geht, hat bessere Noten.

20. Jahrestagung der DGSM 2012

\section{Riskante TV-Vorlieben}

Dermatologen des Brooklyn College, NY, haben 576 Studenten nach ihrem TV-Verhalten befragt. Sie wollten wissen, ob perfekte Körper, wie in Model-TV-Shows zu sehen, bei 18- bis 29-Jährigen eine Begierde nach brauner Haut wecken. Die Unterschiede im Verhalten waren deutlich: 12,9\% der TV-Show-Fans gehen ins Solarium und $43,3 \%$ gaben an, mehr als zwei Stunden am Stück im Freien zu sonnen. Bei den Model-Show-Abstinenzlern waren es nur $3,7 \%$ und $28,7 \%$ an Sonnenanbetern. matol 2012 (online first)

Gilt leider nur für Greise ...

\title{
Wer laufen kann, wird nicht dement!
}

Solange man mit über 90 Jahren körperlich noch fit ist, muss man sich kaum vor einer Demenz fürchten.

In der 90+-Studie ging man dem Zusammenhang von körperlichem und geistigem Abbau im hohen Alter nach: Insgesamt 629 Menschen, im Durchschnitt 94 Jahre, konnten die US-Neurologen um Dr. Szofia Bullain für ihre Untersuchung gewinnen.

\section{0-fach erhöhte Demenzrate}

In der Studie stieg die Demenzrate linear mit der Gebrechlichkeit der Teilnehmer an, und zwar auch dann, wenn bekannte Risikofaktoren wie Schlaganfall und TIA berücksichtigt wurden. In der Studie gab es mehrere Übungen zu absolvieren: eine Wegstrecke $(4 \mathrm{~m})$ laufen, fünfmal von einem Stuhl aufstehen, die Füße hintereinander stellen; zudem wurde die Kraft beim
Zugreifen gemessen. Schon geringfügige körperliche Einschränkungen bei den Uralten gingen mit einem drastisch erhöhten Demenzrisiko einher: ein Versagen bei der Vier-Meter-Strecke ließ die Wahrscheinlichkeit einer Demenz um das 30-fache ansteigen, 15-fach, wer den Stuhl nicht schaffte. Und um den Faktor zehn bewegten sich jeweils die Unterschiede beim Gleichgewichts- und Krafttest.

Da beim Gehen Motorik, Sensorik und Kleinhirn eng zusammenarbeiten, könnten sich durch eine Neurodegeneration bedingte Störungen hier früher bemerkbar machen als bei der Kognition, vermuten die Forscher. Auch, dass der physische Abbau, zu körperlicher Inaktivität führend, den kognitiven Abbau beschleunigt, ist laut der Autoren denkbar. (mut)

Bullain SS et al, JAMA Neurol 2013, 70(1):107-113

\section{Öfter mal die Stelle wechseln}

\section{Insulinspritzen lassen Fett wuchern}

Wer Insulin spritzen muss, entwickelt häufig eine Lipohypertrophie in der Injektionsgegend. Das stört nicht nur kosmetisch, sondern beeinträchtigt auch die Einstellung.

Von 450 Typ-1-Diabetikern, die in einer indischen Studie auf kutane Nebenwirkungen von Insulininjektionen untersucht wurden, wiesen 41,6\% der Probanden eine Lipohypertrophie (LH) auf. Fettwucherungen waren mit einer längeren Dauer der Stoffwechselkrankheit assoziiert, und auch die HbAlc-Werte lagen höher als bei Patienten ohne LH.

Die Hautveränderungen sind oft asymmetrisch, da das Injizieren mit der dominanten Hand eine Körperseite bevorzugt.
Zur Entwicklung trägt weniger die Dosis als vielmehr die Häufigkeit der Injektionen bei. Die lipohypertrophe Region ist relativ schmerzunempfindlich, was sie für die $\mathrm{Pa}$ tienten als Ort, die Spritze zu setzen, attraktiv macht. Allerdings ist auch die Blutversorgung schlecht und es ist nicht vorherzusehen, wie gut das Insulin absorbiert wird. Das führt zu mangelhaft kontrollierter Glykämie, sichtbar am hohen HbAlc. Und dies wiederum hat mikro- und makrovaskuläre Folgen auch an Haut und Gelenken. Durch Wechsel der Injektionsstellen, Verwendung frischer Nadeln und regelmäßiges Absuchen auf kutane Veränderungen ließen sie sich vermeiden. (rb)

Sawatkar GU et al,EADV 2013; doi:10.1111/jdv. 12068 